Philipp Louis

Manufacturing Execution Systems 
GABLER EDITION WISSENSCHAFT 
Philipp Louis

\section{Manufacturing \\ Execution Systems}

Grundlagen und Auswahl

Mit einem Geleitwort von Prof. Dr. Paul Alpar 
Bibliografische Information der Deutschen Nationalbibliothek

Die Deutsche Nationalbibliothek verzeichnet diese Publikation in der

Deutschen Nationalbibliografie; detaillierte bibliografische Daten sind im Internet über $<$ http://dnb.d-nb.de > abrufbar.

Dissertation Universität Marburg, 2008

\section{Auflage 2009}

Alle Rechte vorbehalten

(C) Gabler I GWV Fachverlage GmbH, Wiesbaden 2009

Lektorat: Frauke Schindler / Nicole Schweitzer

Gabler ist Teil der Fachverlagsgruppe Springer Science+Business Media.

www.gabler.de

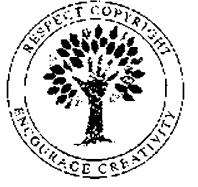

Das Werk einschließlich aller seiner Teile ist urheberrechtlich geschützt. Jede Verwertung außerhalb der engen Grenzen des Urheberrechtsgesetzes ist ohne Zustimmung des Verlags unzulässig und strafbar. Das gilt insbesondere für Vervielfältigungen, Übersetzungen, Mikroverfilmungen und die Einspeicherung und Verarbeitung in elektronischen Systemen.

Die Wiedergabe von Gebrauchsnamen, Handelsnamen, Warenbezeichnungen usw. in diesem Werk berechtigt auch ohne besondere Kennzeichnung nicht zu der Annahme, dass solche Namen im Sinne der Warenzeichen- und Markenschutz-Gesetzgebung als frei zu betrachten wären und daher von jedermann benutzt werden dürften.

Umschlaggestaltung: Regine Zimmer, Dipl.-Designerin, Frankfurt/Main

Gedruckt auf säurefreiem und chlorfrei gebleichtem Papier

Printed in Germany

ISBN 978-3-8349-1018-9 


\section{Geleitwort}

Manufacturing Execution Systeme (MES) stellen eine neue Klasse von Informationssystemen dar, die sich in wachsendem Maße in der produzierenden Industrie etablieren. Bisher beschränkte sich die IT-Unterstützung von Produktionsprozessen maßgeblich auf die Unterstützung von Einzelaktivitäten wie die Produktionsplanung. Auch neuere Ansätze wie die Betrachtung von Planungsaktivitäten über die gesamte Wertschöpfungskette mit Hilfe von Systemen für Supply Chain Management erweitern den Fokus lediglich intra- resp. interorganisatorisch, beziehen aber nicht sämtliche Tätigkeiten innerhalb der Produktion mit ein. Die flexible Steuerung der Produktionsprozesse, etwa in Form eines elektronischen Leitstands, blieb eher eine Vision als gelebte Wirklichkeit.

MES hingegen haben das Ziel, direkt und zeitnah Produktionsprozesse durch den integrierten Einsatz eines oder einiger weniger Informationssysteme flexibel zu unterstützen und so systematische Verbesserungen zu ermöglichen. Zwischen der zunehmenden Nutzung der MES und ihrer methodischen wissenschaftlichen Erörterung und Fortentwicklung tut sich jedoch eine Lücke auf. Erste Schritte, diese zu schließen, sind ebenso Teil der Zielsetzung dieser Arbeit wie die Bereitstellung eines Ansatzes zur Auswahl geeigneter MES-Lösungen für die industrielle Praxis. Dafür werden die bisher im Schrifttum gebräuchlichen, häufig stark industriespezifischen Definitionen vereinheitlicht und daraus ein Framework erarbeitet, das die Anforderungen an ein MES auf die Charakteristika des jeweiligen Produktionssystems zurückführt. Dieser Ansatz konnte in einer empirischen Untersuchung in der deutschen Arzneimittelindustrie, die, nicht zuletzt wegen gesetzlicher Auflagen, hier zu Vorreitern bei der Anwendung von MES gehört, überprüft werden.

Die vorliegende Arbeit kann einen guten Dienst bei der weiteren Entwicklung und Verbreitung von MES leisten, mit denen weitere Fortschritte bei der Flexibilität, Effizienz und Qualitätssicherung in der Produktion erreicht werden können, die ein Hochtechnologiestandort wie Deutschland benötigt, um seine Wettbewerbsfähigkeit zu verteidigen und auszubauen.

Prof. Dr. Paul Alpar 


\section{Vorwort}

Diese Arbeit entstand während meiner Tätigkeit als wissenschaftlicher Mitarbeiter am Lehrstuhl für Wirtschaftsinformatik und quantitative Methoden am Insitut für Wirtschaftsinformatik der Philipps-Universität Marburg und der praktischen Tätigkeit in der Abteilung Information Solutions der CSL Behring GmbH, Marburg.

An erster Stelle möchte ich meinem akademischen Lehrer Prof. Dr. Paul Alpar für die diskursive Unterstützung meiner Promotion und die Schaffung einer angenehmen Arbeitsumgebung danken. Ich wünsche jedem Doktoranden einen Doktorvater, der einen Rahmen für die Promotion schafft und trotzdem so viel Flexibilität in der Ausführung der Arbeit zulässt und sich jede Zeit für Diskussionen und Fragen nimmt.

Meinen Kollegen bei CSL Behring möchte ich für die angenehme und kollegiale Arbeitsatmosphäre danken. Vor allem meinem direkten Vorgesetzten, Herrn Dr. Christoph Kraus, danke ich für sein Engagement, seine fachliche Weitsicht und seine weit über das beruflich vorgegebene Mass hinausgehende Unterstützung meines Promotionsprojektes.

Ein besonderer Dank geht an Herrn Prof. Dr. Ulrich Hasenkamp für die Übernahme der Zweitkorrektur und die trotz seiner vielfältigen Verpflichtungen überaus schnelle Erstellung des Zweitgutachtens, die mir eine Anreise von weit her zur Disputation erspart hat. Weiterhin danke ich den Herren Prof. Dr. Bernd Hayo und Prof. Dr. Stefan Dirkes für die Durchsicht der Arbeit und die Durchführung der Disputation.

Mein größter Dank geht an dieser Stelle an meine ganze Familie, die mich auf meinem Lebensweg stets liebevoll unterstützt hat. Vor allem meiner Frau Janina und meinem (Doktor-)Vater gebührt hier ein besonderer Dank. Janina, vielen Dank dass Du mich immer (wenn auch zeitweise unkonventionell) motiviert und unterstützt hast - ich liebe Dich und Du bist der Mittelpunkt meines Lebens! Papa, Dir möchte ich danken für das Engagement vieler Stunden, in denen Du mit mir methodische wie inhaltliche Punkte kritisch erörtert und mitunter näher auf den Punkt gebracht hast. 


\section{Inhaltsverzeichnis}

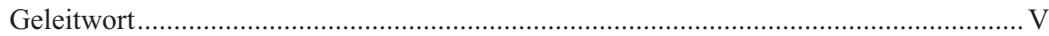

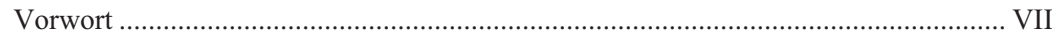

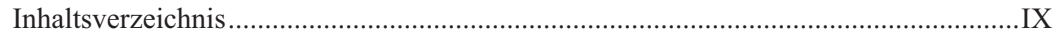

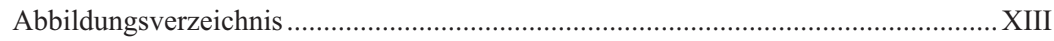

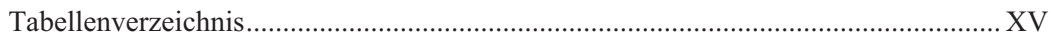

Abkürzungsverzeichnis ................................................................................... XVII

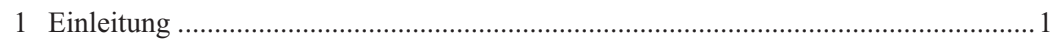

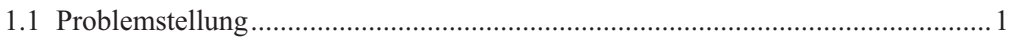

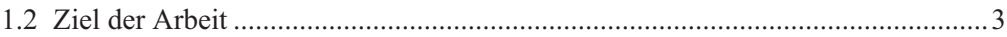

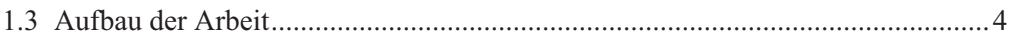

2 Manufacturing Execution Systeme (MES) ........................................................... 7

2.1 Bisherige MES-Begriffsdefinitionen.......................................................... 7

2.1.1 MES-Definition der Manufacturing Enterprise Solutions Association ....... 8

2.1.2 MES-Definition der Instrumentations, Systems, and Automation Society 12

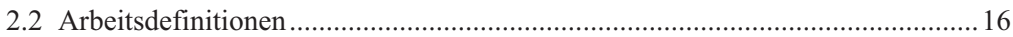

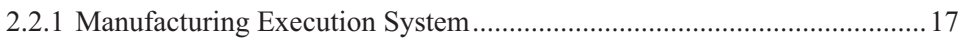

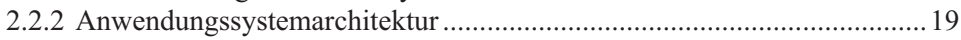

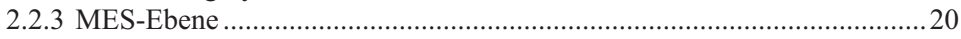

2.3 Funktionsgruppen der MES-Ebene ..................................................................... 21

2.3.1 Production Operation Management (POM) ….......................................24

2.3.2 Maintenance Operation Management (MOM) ........................................26

2.3.3 Quality Operation Management (QOM) ..................................................29

2.3.4 Inventory Operation Management (IOM) .............................................. 31

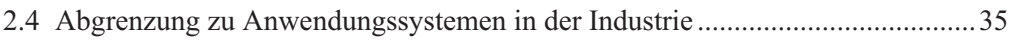

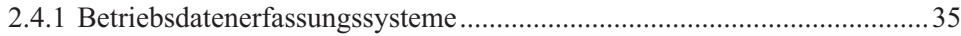

2.4.2 Produktionsplanungs- und Steuerungssysteme ..................................... 37

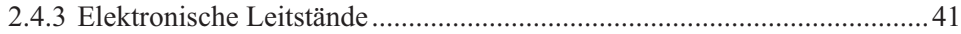

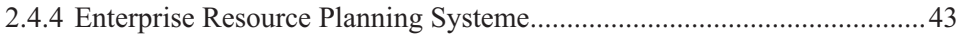

2.4.5 CIM-Einordnung der Anwendungssysteme............................................. 46

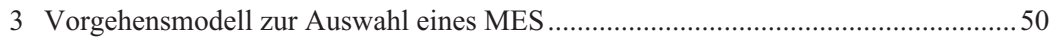

3.1 Charakterisierung der Produktionsprozesse ...........................................................5 53

3.2 Ableitung von Anforderungen an die MES-Ebene ...............................................5 54

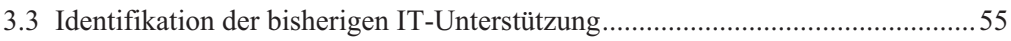

3.4 Szenariodefinition, -bewertung und -auswahl ............................................... 57

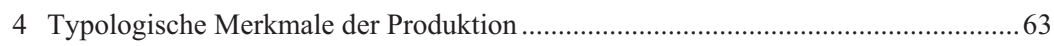

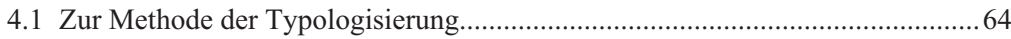

4.1.1 Definition des Untersuchungszieles und des Untersuchungsbereiches .....6 64

4.1.2 Auswahl der zu untersuchenden Merkmale .............................................65

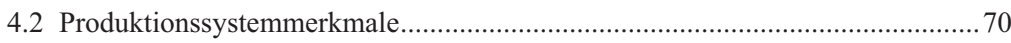

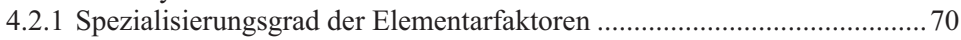

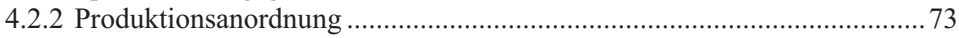

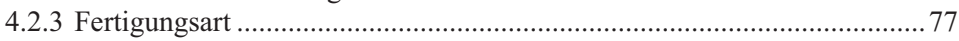




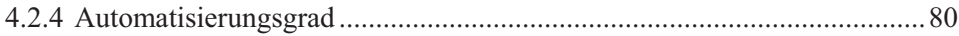

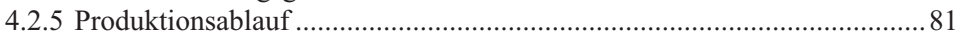

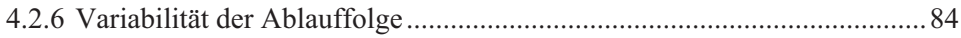

4.2.7 Betriebsmittel- und Prozesssubstituierbarkeit..............................................86

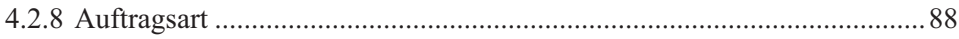

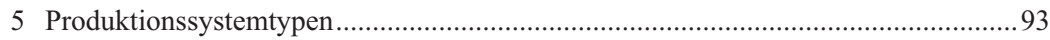

5.1 Zur Bildung von Verbundtypen...................................................................93

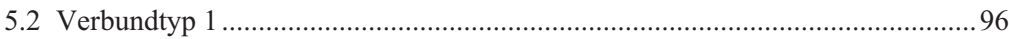

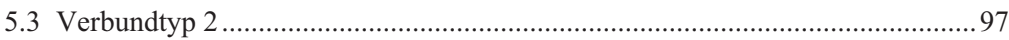

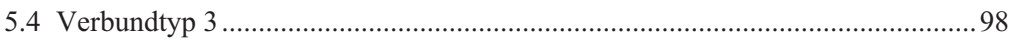

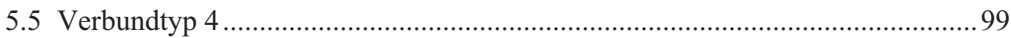

6 Zur Ableitung von Anforderungen an die MES-Ebene.............................................. 100

6.1 Auswirkungen der Merkmale auf die Teilbereiche der MES-Ebene ..................100

6.1.1 Production Operation Management ......................................................... 101

6.1.2 Maintenance Operation Management ........................................................ 102

6.1.3 Quality Operation Management................................................................ 103

6.1.4 Inventory Operation Management ………………………………........... 105

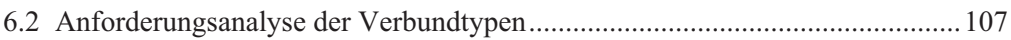

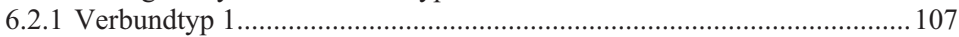

6.2.1.1 Anforderungen an das Production Operation Management..........107

6.2.1.2 Anforderungen an das Maintenance Operation Management...... 110

6.2.1.3 Anforderungen an das Quality Operation Management ...............112

6.2.1.4 Anforderungen an das Inventory Operation Management............114

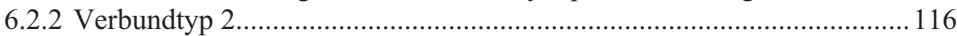

6.2.2.1 Anforderungen an das Production Operation Management..........116

6.2.2.2 Anforderungen an das Maintenance Operation Management......119

6.2.2.3 Anforderungen an das Quality Operation Management ...............121

6.2.2.4 Anforderungen an das Inventory Operation Management............123

6.2.3 Verbundtyp 3 …………………………………………………. 125

6.2.3.1 Anforderungen an das Production Operation Management..........125

6.2.3.2 Anforderungen an das Maintenance Operation Management......127

6.2.3.3 Anforderungen an das Quality Operation Management ...............130

6.2.3.4 Anforderungen an das Inventory Operation Management............131

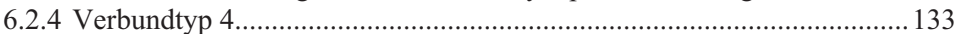

6.2.4.1 Anforderungen an das Production Operation Management..........133

6.2.4.2 Anforderungen an das Maintenance Operation Management.......135

6.2.4.3 Anforderungen an das Quality Operation Management ...............137

6.2.4.4 Anforderungen an das Inventory Operation Management............139

6.3 Vorgehen bei Kombinationen von Verbundtypen............................................. 141

7 Empirische Überprüfung der Ableitung von Anforderungen..................................... 144

7.1 Rahmenbedingungen innerhalb der pharmazeutischen Industrie......................... 144

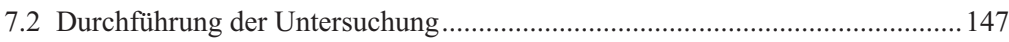

7.3 Zur aktuellen Unterstützung der MES-Ebene ................................................... 148

7.3.1 Ergebnisse im Teilbereich Production Operation Management ............... 151

7.3.2 Ergebnisse im Teilbereich Maintenance Operation Management ............153

7.3.3 Ergebnisse im Teilbereich Quality Operation Management.......................155

7.3.4 Ergebnisse im Teilbereich Inventory Operation Management ...................157

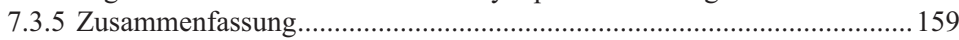


7.4 Überprüfung der Ergebnisse der typologischen Ableitung .............................. 160

7.4.1 Überprüfung des Teilbereichs POM ...................................................... 162

7.4.2 Überprüfung des Teilbereichs MOM .................................................. 164

7.4.3 Überprüfung des Teilbereichs QOM................................................... 165

7.4.4 Überprüfung des Teilbereichs IOM ..................................................... 166

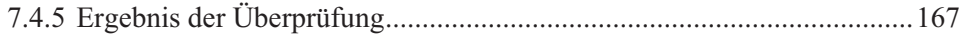

8 Bewertung der Kosten von MES-Szenarien...................................................... 168

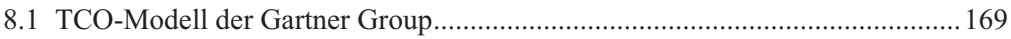

8.2 TCO-Modell zur Bewertung von Anwendungssystemen................................. 172

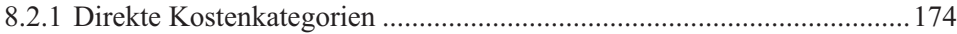

8.2.2 Indirekte Kostenkategorien .............................................................. 176

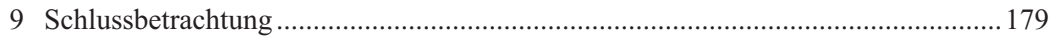

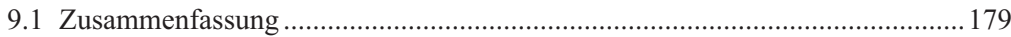

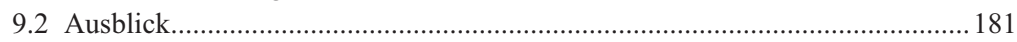

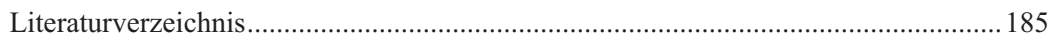

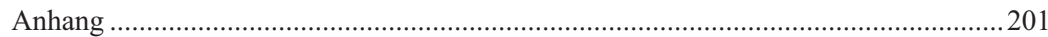

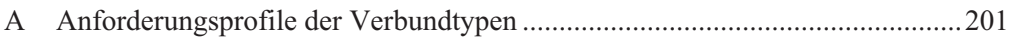

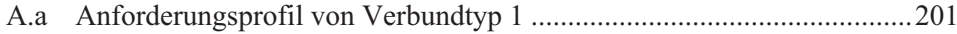

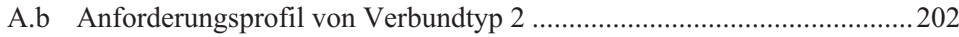

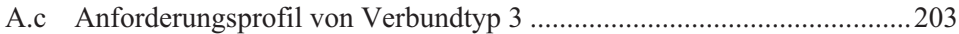

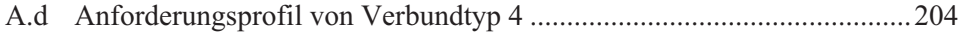

B Fragebogen der empirischen Untersuchung ..................................................206 


\section{Abbildungsverzeichnis}

Abb. 1.1: Vorgehensweise der Arbeit ............................................................................. 6

Abb. 2.1: MES als Verbund der 11 MESA-Funktionen............................................... 11

Abb. 2.2: Geltungsbereich des Purdue Reference Model .......................................... 13

Abb. 2.3: Hierarchisches Modell des S95 Standards ................................................. 14

Abb. 2.4: MES-ERP-Grenze des S95 Standards ......................................................... 15

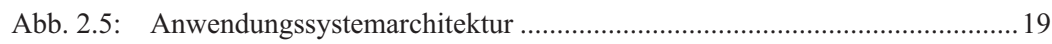

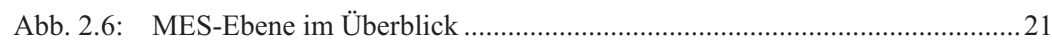

Abb. 2.7: Teilbereiche der MES-Ebene ….................................................................. 22

Abb. 2.8: Generisches Funktionsgruppenmodell der MES-Ebene ..............................2 23

Abb. 2.9: Funktionsgruppen des Production Operation Managements.........................24

Abb. 2.10: Funktionsgruppen des Maintenance Operation Managements .....................27

Abb. 2.11: Funktionsgruppen des Quality Operation Managements ............................. 30

Abb. 2.12: Funktionsgruppen des Inventory Operation Managements............................ 32

Abb. 2.13: Stufenmodell des MRP II-Konzeptes .............................................................. 38

Abb. 2.14: Überschneidung der Aufgaben von PPS-Systemen und MES ..................... 39

Abb. 2.15: Graphische Darstellung der Arbeitsplatzplanung........................................... 41

Abb. 2.16: Funktionsumfang von mySAP ERP von SAP .......................................... 43

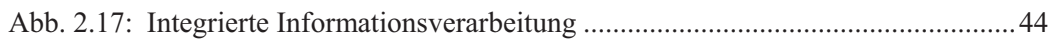

Abb. 2.18: Eingeführte Anwendungssysteme im Y-CIM Modell...................................47

Abb. 3.1: Abgedeckte Phasen des generischen Vorgehensmodells nach Grochla.......51

Abb. 3.2: Vorgehensmodell zur Auswahl eines MES ................................................... 53

Abb. 3.3: Exemplarisches Ergebnis von Phase 2 (nur POM) ......................................... 55

Abb. 3.4: Exemplarisches Ergebnis von Phase 3 (nur POM) ....................................... 56

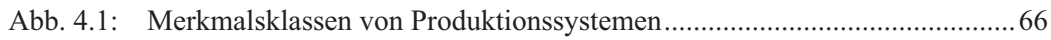

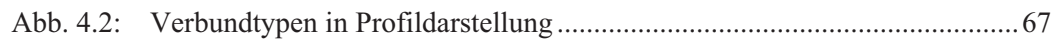

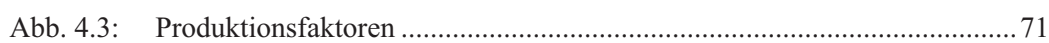

Abb. 4.4: Flexibilität und Produktivität der Maschinentypen ....................................... 72

Abb. 4.5: Kontinuierlicher und diskontinuierlicher Materialfluss ................................ 83

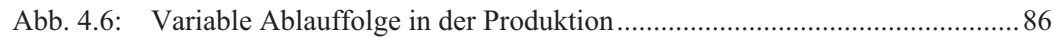

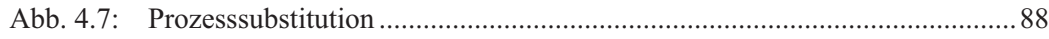

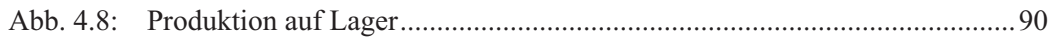

Abb. 6.1: Anforderungen des Verbundtyps 1 an das POM ...................................... 110

Abb. 6.2: Anforderungen des Verbundtyps 1 an das MOM ...................................... 112

Abb. 6.3: Anforderungen des Verbundtyps 1 an das QOM ........................................ 114

Abb. 6.4: Anforderungen des Verbundtyps 1 an das IOM........................................ 116 
Abb. 6.5: Anforderungen des Verbundtyps 2 an das POM....................................... 119

Abb. 6.6: Anforderungen des Verbundtyps 2 an das MOM ...................................... 121

Abb. 6.7: Anforderungen des Verbundtyps 2 an das QOM ....................................... 123

Abb. 6.8: Anforderungen des Verbundtyps 2 an das IOM......................................... 125

Abb. 6.9: Anforderungen des Verbundtyps 3 an das POM...................................... 127

Abb. 6.10: Anforderungen des Verbundtyps 3 an das MOM ...................................... 129

Abb. 6.11: Anforderungen des Verbundtyps 3 an das QOM ...................................... 131

Abb. 6.12: Anforderungen des Verbundtyps 3 an das IOM........................................ 133

Abb. 6.13: Anforderungen des Verbundtyps 4 an das POM.......................................... 135

Abb. 6.14: Anforderungen des Verbundtyps 4 an das MOM ...................................... 137

Abb. 6.15: Anforderungen des Verbundtyps 4 an das QOM ....................................... 139

Abb. 6.16: Anforderungen des Verbundtyps 4 an das IOM........................................ 141

Abb. 7.1: Positionen der Interviewpartner .............................................................. 147

Abb. 7.2: Durchschnittliche Anforderungen an die MES-Ebene und deren aktuelle Unterstützung durch derzeitige Anwendungssysteme ............................. 149

Abb. 7.3: Anforderungen und aktuelle Unterstützung des POM ............................... 152

Abb. 7.4: Anforderungen und aktuelle Unterstützung des MOM ............................... 154

Abb. 7.5: Anforderungen und aktuelle Unterstützung des QOM................................ 156

Abb. 7.6: Anforderungen und aktuelle Unterstützung des IOM ............................... 158

Abb. 7.7: Verbundtypenzuordnung und deren Abweichungen ................................... 161

Abb. 7.8: Empirische und abgeleitete Anforderungen an das POM .......................... 163

Abb. 7.9: Empirische und abgeleitete Anforderungen an das MOM........................... 164

Abb. 7.10: Empirische und abgeleitete Anforderungen an das QOM........................... 165

Abb. 7.11: Empirische und abgeleitete Anforderungen an das IOM ............................ 166

Abb. 8.1: Grundsätzliche Aufteilung von TCO .......................................................... 168

Abb. 8.2: Kostenkategorien des TCO-Modells der Gartner Group ............................. 170

Abb. 9.1: Vorgehensmodell zur Auswahl eines MES ................................................. 180 


\section{Tabellenverzeichnis}

Tab. 2.1: Teilbereiche und Funktionen der MES-Ebene ............................................... 34

Tab. 3.1: Exemplarisches Ergebnis von Phase 1 …........................................................ 54

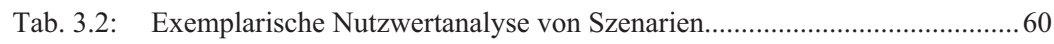

Tab. 3.3: Exemplarische Nutzwertanalyse nach Sensitivitätsanalyse .........................62 62

Tab. 4.1: Auswirkungen der Leitmerkmale auf Auftragsveranlassung und

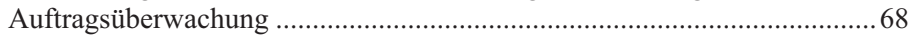

Tab. 4.2: Wirkung der Merkmale auf die Teilbereiche der MES-Ebene.......................69

Tab. 4.3: Ausgewählte Merkmale der Typologisierung ............................................... 70

Tab. 4.4: Elementartypenreihe Spezialisierungsgrad der Elementarfaktoren .............. 73

Tab. 4.5: Elementartypenreihe Produktionsanordnung .............................................. 77

Tab. 4.6: Elementartypenreihe Fertigungsart ........................................................... 80

Tab. 4.7: Ableitung der Automatisierungsgrade..................................................... 81

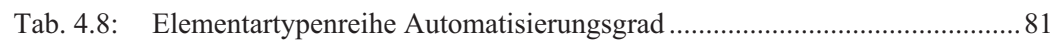

Tab. 4.9: Elementartypenreihe Prozessablauf ................................................................. 84

Tab. 4.10: Elementartypenreihe Variabilität der Ablauffolge ....................................... 86

Tab. 4.11: Elementartypenreihe Betriebsmittel- und Prozesssubstituierbarkeit............. 88

Tab. 4.12: Elementartypenreihe Auftragsart ................................................................ 91

Tab. 4.13: Merkmale mit deren Elementartypenreihen ................................................. 92

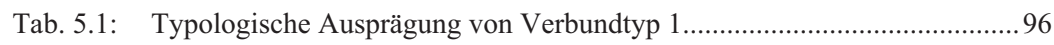

Tab. 5.2: Typologische Ausprägung von Verbundtyp 2 ...........................................97

Tab. 5.3: Typologische Ausprägung von Verbundtyp 3............................................ 98

Tab. 5.4: Typologische Ausprägung von Verbundtyp 4............................................. 99

Tab. 6.1: Auswirkungen der Merkmale auf den POM - Teilbereich.......................... 102

Tab. 6.2: Auswirkungen der Merkmale auf den MOM - Teilbereich ......................... 103

Tab. 6.3: Auswirkungen der Merkmale auf den QOM - Teilbereich .......................... 104

Tab. 6.4: Auswirkungen der Merkmale auf den IOM - Teilbereich............................ 106

Tab. 6.5: Auswirkungen der Merkmale auf die Teilbereiche der MES-Ebene .......... 106

Tab. 6.6: Kombination von Anforderungsprofilen ....................................................... 142

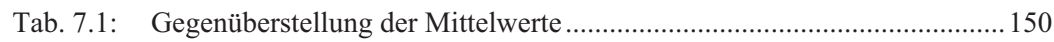

Tab. 7.2: Korrelation zwischen allgemeiner und errechneter Bewertung .................. 151

Tab. 7.3: Unterschiede zwischen Anforderung und Unterstützung des POM........... 153

Tab. 7.4: Unterschiede zwischen Anforderung und Unterstützung des MOM ......... 155

Tab. 7.5: Unterschiede zwischen Anforderung und Unterstützung des QOM .......... 157

Tab. 7.6: Unterschiede zwischen Anforderung und Unterstützung des IOM............. 158

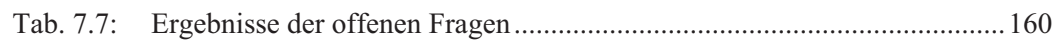


Tab. 7.8: Empirische und abgeleitete Anforderungen an die MES-Ebene................. 162

Tab. 8.1: TCO Kostenkategorien eines MES .......................................................... 173 


\section{Abkürzungsverzeichnis}

\begin{tabular}{|c|c|}
\hline BDE & Betriebsdatenerfassung \\
\hline BFS & Betriebsführungssystem \\
\hline BPR & Business Process Reengineering \\
\hline B2MML & Business to Manufacturing Markup Language \\
\hline CAP & Computer Aided Planning \\
\hline CFR & Code of Federal Regulation \\
\hline $\begin{array}{l}\text { CIM } \\
\text { et al. }\end{array}$ & $\begin{array}{l}\text { Computer Integrated Manufacturing } \\
\text { et alii }\end{array}$ \\
\hline ERP & Enterprise Resource Planning \\
\hline FDA & Food and Drug Administration \\
\hline GMP & Good Manufacturing Practice \\
\hline IOM & Inventory Operation Management \\
\hline IS & Informationssystem \\
\hline ISA & Instrumentations, Systems, and Automation Society \\
\hline IT & Informationstechnologie \\
\hline KPI & Key Performance Indicator \\
\hline KVP & kontinuierlicher Verbesserungsprozess \\
\hline LIMS & Laboratory Information Management System \\
\hline MDE & Maschinendatenerfassung \\
\hline MES & Manufacturing Execution System \\
\hline MESA & Manufacturing Enterprise Solutions Association \\
\hline MOM & Maintenance Operation Management \\
\hline MRP II & Manufacturing Resource Planning \\
\hline NAMUR & $\begin{array}{l}\text { Normenarbeitsgemeinschaft für Mess- und Regeltechnik in der chemischen } \\
\text { Industrie }\end{array}$ \\
\hline OMS & Operation Management System \\
\hline $\mathrm{OPC}$ & OLE for Process Control \\
\hline PAC & Production Activity Control \\
\hline PAT & Process Analytical Technology \\
\hline POM & Production Operation Management \\
\hline PPS & Produktionsplanung und -steuerung \\
\hline PRM & Purdue Reference Model \\
\hline PZE & Personalzeiterfassung \\
\hline QOM & Quality Operation Management \\
\hline $\mathrm{RAC}$ & Real Application Cluster \\
\hline RFID & Radio Frequency Identification \\
\hline SAP & Systeme, Anwendungen, Produkte in der Datenverarbeitung \\
\hline SCM & Supply Chain Management \\
\hline SLA & Service Level Agreement \\
\hline SOA & Service oriented architecture \\
\hline TCO & Total cost of ownership \\
\hline TQM & Total quality management \\
\hline WBF & World Batch Forum \\
\hline WIP & Work in Progress \\
\hline VDI & Verein deutscher Ingenieure \\
\hline
\end{tabular}

